\title{
Embolic infarction followed by serial bone SPECT and MR fusion images -the door to SPECT/MR-
}

\author{
Tetsuya Tsujikawa, Tomohito Kamibayashi, Tatsuro Tsuchida, Yusuke Ohkoshi, \\ and Hirohiko Kimura \\ Department of Radiology, University of Fukui, Fukui, Japan
}

\begin{abstract}
We recently experienced a case of cerebral infarction incidentally found by whole body bone scintigraphy for the detection of bone metastasis from renal cell carcinoma. Additional bone SPECT and brain MR fusion images clearly demonstrated the wedge-shaped uptake of tracer corresponded to the abnormal intensity reflecting subacute cerebral infarction. Follow-up bone scan and fused images with MRI showed complete resolution of the abnormal uptake in chronic phase. A breakdown in the normal blood-brain barrier results in abnormal ionic calcium flux into the cells following altered cell membrane integrity leading to precipitation of calcium salts which eventually binds to bone imaging tracer such as ${ }^{99 m}$ Tc-methylene diphosphonate. That is, increased accumulation of bone seeking agents represents lethal cell death. The recent development of software and hardware has enabled the fusion of functional and anatomic images. Image fusion between SPECT with various tracers and MRI is expected to provide clues as to the underlying cause of diseases and to decide our treatment planning in the near future. J. Med. Invest. 57 : 334-337, August, 2010
\end{abstract}

Keywords : bone SPECT, MRI, fusion, functional and anatomic images

\section{INTRODUCTION}

Bone scintigraphy is mainly used for the detection of bone metastasis in patients with known malignancy, in which extra-osseous abnormal accumulations can be seen coincidentally. Bone imaging tracer such as ${ }^{99 \mathrm{~m}} \mathrm{Tc}$-methylene diphosphonate is taken up by cerebral infarction (1) and myocardial infarction along with soft tissue calcification, infection and inflammation. In addition, the recent development of software and hardware has enabled the fusion of functional and anatomic images. We report a 54-year-old man with cerebral infarctions

Received for publication January 5, 2010 ; accepted February $10,2010$.

Address correspondence and reprint requests to Tetsuya Tsujikawa, Department of Radiology, University of Fukui, Fukui, Japan, 23-3 Matsuoka-Shimoaizuki, Eiheiji-cho, Fukui 9101193, Japan and Fax : +81-776-61-8137. longitudinally followed by bone SPECT and brain MR fusion images. To our knowledge, our report is first one in which cerebral infarctions were longitudinally followed by the fusion images.

\section{CASE REPORT}

A 54-year-old man with persistent fever due to infectious endocarditis was referred to our hospital. He had no history of any medical illness and had not undergone surgery. Abdominal CT scan obtained after admission happened to depict left renal cell carcinoma (RCC) after which he underwent bone scintigraphy with ${ }^{99 m}$ Tc-methylene diphosphonate (99m Tc-MDP) for detecting metastatic lesions. Anterior planar image showed longitudinal strong uptake of tracer in left frontal region of head (Fig. 1A). Because the uptake was not characteristic 

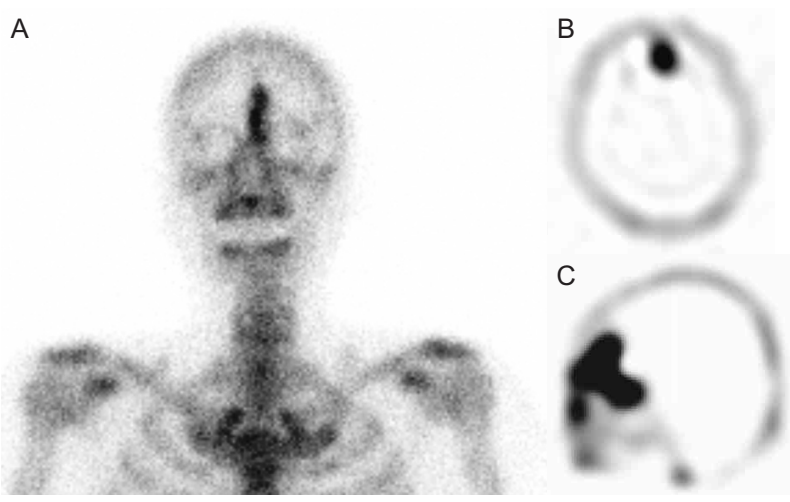

Figure 1. Bone scintigraphy (A) demonstrates longitudinal strong uptake of ${ }^{99 \mathrm{~m} T c}$-methylene diphosphonate in left frontal region of head. Bone SPECT images $(\mathrm{B}, \mathrm{C})$ indicate that the uptake exists in left frontal lobe.

of bone metastasis additional SPECT scans were added, which revealed that the uptake existed in left frontal lobe (Fig. 1B, C). Brain MRI examined on the following day showed slightly hyper-intense signal in the lesion on diffusion-weighted (DWI) (Fig. 2A), T2-weighted and fluid-attenuated inversionrecovery (FLAIR) (Fig. 2B) images which suggested

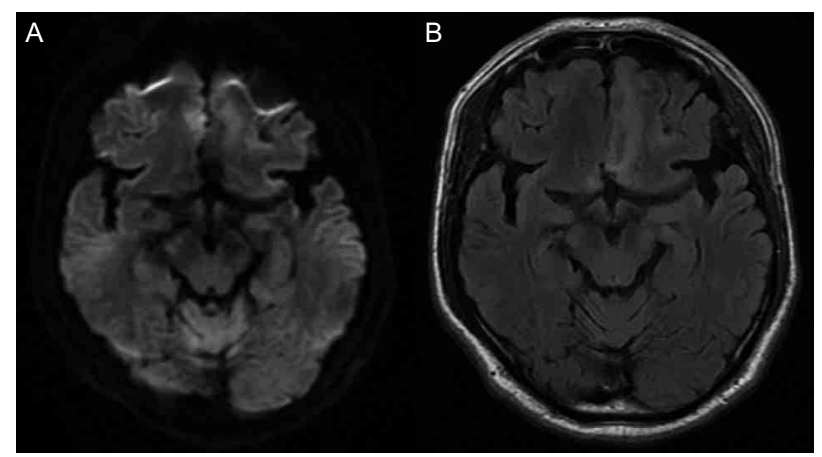

Figure 2. Axial fluid-attenuated inversion-recovery (A) and diffusion-weighted (B) MR images demonstrate slightly hyperintense signal in left frontal lobe which suggest subacute embolic infarcts due to endocarditis with pseudo-normalization.

subacute embolic infarcts due to endocarditis with mild degree of pseudo-normalization. Brain MRI performed two weeks before bone scintigraphy had indicated almost no abnormality. Bone SPECT and MR images were readily fused by use of software (Fusion Viewer version 1.5, Nihon MediPhysics Co. Ltd., Nishinomiya, Japan). Abnormal uptake of tracer on bone SPECT perfectly corresponded with abnormal intensity on FLAIR image (Fig. 3). Sagittal view of the fusion images clearly delineated a wedge-shaped lesion which corresponds to left anterior cerebral artery (ACA ; A2-3) territory (Fig. 3C). Follow-up MR exams in the

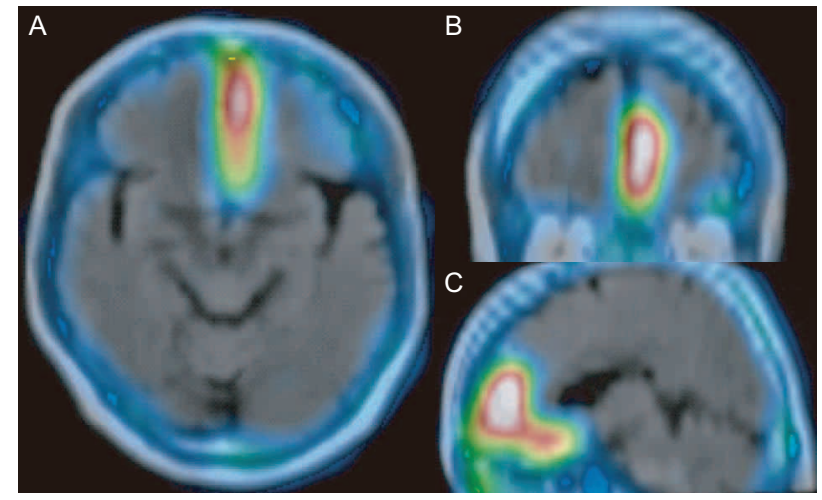

Figure 3. Bone SPECT and FLAIR fused images show that abnormal uptake of $99 \mathrm{mTc}-\mathrm{MDP}$ perfectly corresponds with abnormal intensity on FLAIR images. A : axial image, B : coronal image, $\mathrm{C}$ : sagittal image.

chronic phase showed sharply-defined T2-high intensity and perfusion decrease. (not shown)

Three months later, he underwent bone scintigraphy again as preoperative study for detecting bone metastasis from RCC. The bone scan and fused images with MRI showed complete resolution of the abnormal uptake in left anterior lobe (Fig. 4, 5).

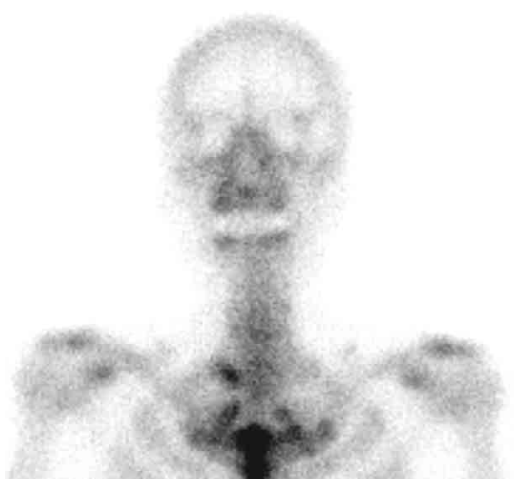

Figure 4. Bone scintigraphy on chronic phase shows complete resolution of the abnormal uptake in left anterior lobe.

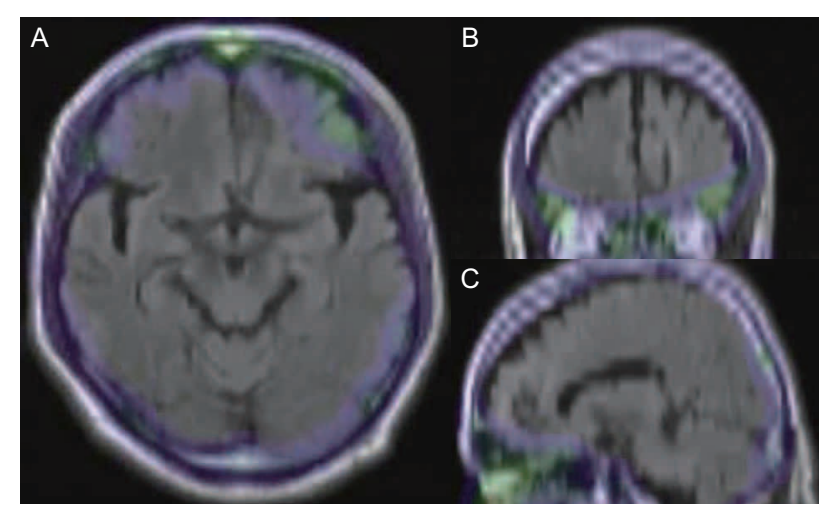

Figure 5. Bone SPECT and FLAIR fused images in chronic phase also show complete resolution of the abnormal uptake in left anterior lobe. 


\section{DISCUSSION}

Incidental cerebral uptake of bone imaging agents has been found in $0.17 \%$ of bone scans and in most cases is the result of intracranial malignancy (2). Extra-osseous uptake of bone imaging agents in cerebral infarction has been reported sporadically (3-8). A breakdown in the normal blood-brain barrier results in abnormal ionic calcium flux into the cells following altered cell membrane integrity leading to precipitation of calcium salts which eventually binds to ${ }^{99 m}$ Tc-phosphate. Additional bone SPECT and brain MR fusion images combined molecular information with anatomic one which enabled the case to be diagnosed as subacute embolic infarction of left ACA territory. In addition, follow-up fusion images clearly showed the disappearance of ${ }^{99 \mathrm{~m}} \mathrm{Tc}$ MDP accumulation in chronic phase (after three months of onset). There has been no literature describing in detail the critical period of the appearance and the disappearance of bone seeking tracer accumulation in cerebral infarct. Short interval follow-up exams using bone imaging tracer are difficult in practice, whereas bone scintigraphy can probably demonstrate cerebral infarcts as increased uptake in acute or subacute phase (within 18 days of onset) and as normalized one in chronic phase (after the 6th week of onset) based on previous case reports (3-8).

Molecular and anatomic image fusion has widely spread in recent years with the development of fusion software and hardware such as PET/CT and SPECT/CT scanners (9). In addition, combined PET/MR scanners have only recently been developed, and several MRI-based attenuation correction methods have been reported to obtain quantitative PET information $(10,11)$. MR images provide not only anatomic information but also abnormal intensity reflecting restricting microscopic proton diffusion [apparent diffusion coefficient (ADC) decrease] and $\mathrm{T} 2$ elongation of the lesion. The subtle ADC decrease seen in reversible lesions like transient ischemic attack (TIA) may correspond to a mild ischemia that does not cause advanced bioenergetic failure and subsequent permanent tissue injury $(12,13)$. Lesions detected by DWI with similar $\mathrm{ADC}$ changes have also been reported in association with reversal of DWI hyperintensities after intraarterial thrombolysis in acute stroke patients (14). On the other hand, increased accumulation of bone seeking agents represents the damage of $\mathrm{Ca}^{2+} / \mathrm{Mg}^{2+}$ ATPase on cell membrane which leads to $\mathrm{Ca}^{2+}$ influx into the cells and consequently lethal cell death.

Software image registration and fusion allow general versatility in the choice of modalities and facilitates image fusion of several modalities which reflect different kinds of molecular biologic characterization $(15,16)$. Uematsu et al. emphasized that normal distribution of FDG uptake in the head and neck regions delineated by multidirectional sections is important for effective co-registration of FDGPET with MRI in their previous report (17). In this case using bone SPECT, tracer accumulation in cranial bones was used for accurate fusion with MR images. Moreover, since there are various kinds of SPECT tracer reflecting the pathophysiological characteristics, the development of hardware like combined SPECT/MR scanner as well as PET/MR scanner are expected to provide clues as to the underlying cause of diseases and to decide our treatment planning in the near future.

\section{REFERENCES}

1. Grames GM, Jansen $\mathrm{C}$ : The abnormal bone scan in cerebral infarction. J Nucl Med 14 : 941-943, 1973

2. Wakisaka M, Miyake H, Hori Y, Takeoka H, Ueno S, Ueda S, Matsumoto S, Monzen Y, Funakoshi $\mathrm{T}$ : Intracranial accumulation of 99mTc-phosphorous compound on bone scintigraphy. Kaku Igaku 28 : 1343-1347, 1991 (in Japanese)

3. Mountz JM, Malinoff H, Wilson MW : Silent infarction of the brain. Incidental finding demonstrated by SPECT. Clin Nucl Med 13 : 498501, 1988

4. Wallace JC, Beyea D : Cerebral infarction pattern identified on emission computed tomography using technetium-99m MDP : Clin Nucl Med 13 : 268-270, 1988

5. Moallem A:Tc-99m MDP appearance and resolution in a cerebral infarct in a patient with dementia. Clin Nucl Med 16 : 676-677, 1991

6. Low RD, Hicks RJ, Gill G, Arkles LB : Tc-99m MDP uptake in a cerebral infarct. Clin Nucl Med 17 : 968-970, 1992

7. Mackie GC:Tc-99m MDP uptake resulting from acute middle cerebral artery territory infarction. Clin Nucl Med $28: 851-852,2003$

8. Crawshaw JW, Allan R: Middle cerebral artery infarct demonstrated on bone scintigraphy. Eur J Nucl Med Mol Imaging 34 : 306, 2007 
9. Gnanasegaran G, Cook G, Adamson K, Fogelman I : Patterns, variants, artifacts, and pitfalls in conventional radionuclide bone imaging and SPECT/CT. Semin Nucl Med 39 : 380395, 2009

10. Hofmann M, Steinke F, Scheel V, Charpiat G, Farquhar J, Aschoff P, Brady M, Schölkopf B, Pichler BJ : MRI-based attenuation correction for PET/MRI : a novel approach combining pattern recognition and atlas registration. J Nucl Med 49 : 1875-1883, 2008

11. Martinez-Möller A, Souvatzoglou M, Delso G, Bundschuh RA, Chefd'hotel C, Ziegler SI, Navab N, Schwaiger M, Nekolla SG : Tissue classification as a potential approach for attenuation correction in whole-body PET/MRI : evaluation with PET/CT data. J Nucl Med 50 : 520-526, 2009

12. Saver JL, Kidwell C : Neuroimaging in TIAs. Neurology 62 : S22-25, 2004

13. Oppenheim C, Lamy C, Touzé E, Calvet D, Hamon M, Mas JL, Méder JF : Do transient ischemic attacks with diffusion-weighted imaging abnormalities correspond to brain infarctions? AJNR Am J Neuroradiol 27 : 1782-1787, 2006

14. Schaefer PW, Hassankhani A, Putman C, Sorensen AG, Schwamm L, Koroshetz W, Gonzalez RG : Characterization and evolution of diffusion MR imaging abnormalities in stroke patients undergoing intra-arterial thrombolysis. AJNR Am J Neuroradiol 25 : 951-957, 2004

15. Slomka PJ : Software approach to merging molecular with anatomic information. J Nucl Med $45:$ 36S-45S, 2004

16. Popovich T, Carpenter JS, Rai AT, Carson LV, Williams HJ, Marano GD : Spinal cord compression by tophaceous gout with fluorodeoxyglucose-positron-emission tomographic/MR fusion imaging. AJNR Am J Neuroradiol 27 : 1201-1203, 2006

17. Uematsu H, Sadato N, Yonekura Y, Tsuchida T, Nakamura S, Sugimoto K, Waki A, Yamamoto K, Hayashi N, Ishii Y : Coregistration of FDG PET and MRI of the head and neck using normal distribution of FDG. J Nucl Med 39 : 21212127, 1998 